\title{
Intestinal-type of differentiation predicts favourable overall survival: confirmatory clinicopathological analysis of 198 periampullary adenocarcinomas of pancreatic, biliary, ampullary and duodenal origin
}

Peter Bronsert ${ }^{1}$, Ilona Kohler ${ }^{1}$, Martin Werner ${ }^{1}$, Frank Makowiec ${ }^{2}$, Simon Kuesters ${ }^{2}$, Jens Hoeppner ${ }^{2}$, Ulrich Theodor Hopt ${ }^{2}$, Tobias Keck ${ }^{2,3}$, Dirk Bausch ${ }^{2,3}$ and Ulrich Friedrich Wellner ${ }^{2,3^{*}}$

\begin{abstract}
Background: Periampullary adenocarcinomas comprise pancreatic, distal bile duct, ampullary and duodenal adenocarcinoma. The epithelia of these anatomical structures share a common embryologic origin from the foregut. With steadily increasing numbers of pancreatoduodenectomies over the last decades, pathologists, surgeons and oncologists are more often confronted with the diagnosis of "other than pancreatic" periampullary cancers. The intestinal subtype of ampullary cancer has been shown to correlate with better prognosis.

Methods: Histological subtype and immunohistochemical staining pattern for CK7, CK20 and CDX2 were assessed for $n=198$ cases of pancreatic ductal, distal bile duct, ampullary and duodenal adenocarcinoma with clinical followup. Routine pathological parameters were included in survival analysis performed with SPSS 20.

Results: In univariate analysis, intestinal subtype was associated with better survival in ampullary, pancreatic ductal and duodenal adenocarcinoma. The intestinal type of pancreatic ductal adenocarcinoma was not associated with intraductal papillary mucinous neoplasm and could not be reliably diagnosed by immunohistochemical staining pattern alone. Intestinal differentiation and lymph node ratio, but not tumor location were independent predictors of survival when all significant predictor variables from univariate analysis (grade, TNM stage, presence of precursor lesions, surgical margin status, perineural, vascular and lymphatic vessel invasion, CK7 and CDX2 staining pattern) were included in a Cox proportional hazards model.
\end{abstract}

Conclusions: Intestinal type differentiation and lymph node ratio but not tumor location are independent prognostic factors in pooled analysis of periampullary adenocarcinomas. We conclude that differentiation is more important than tumor location for prognostic stratification in periampullary adenocarcinomas.

\footnotetext{
* Correspondence: dr.ulrich.wellner@gmail.com

${ }^{2}$ Clinic for General and Visceral Surgery, University of Freiburg, Hugstetter Str.

55, Freiburg 79106, Germany

${ }^{3}$ Clinic for Surgery, University of Schleswig-Holstein Campus Lübeck,

Ratzeburger Allee 160, Lübeck 23582, Germany

Full list of author information is available at the end of the article
} 


\section{Background}

The present WHO classification of tumors distinguishes between pancreatic ductal (PDAC), extrahepatic (distal) bile duct (DBDAC), ampullary (AMPAC) and small intestinal (including duodenal, DUOAC) adenocarcinoma [1]. A fundamental observation is that survival after resection of adenocarcinoma of periampullary location (pancreatic head, distal bile duct, ampulla, duodenum) differs greatly, with DUOAC and AMPAC displaying a much better survival than pancreatic head PDAC or DBDAC, implying several issues of continued debate [2-4].

First, due to the anatomical complexity of the periampullary region, correct classification with respect to location remains challenging to the pathologist. Usually the origin of a periampullary tumor is defined macroscopically by location of the main tumor mass or eventual precursor lesions and has to be confirmed microscopically [2-4]. There is still considerable debate on how localization of adenocarcinomas and their precursor lesions arising in this region should be assessed [2,5].

Another aspect is the question of the biological basis of the observed differences in survival. A major step was the recognition of the intestinal (INT) versus pancreatobiliary (PB) histopathologic phenotypes of AMPAC by Kimura et al. in 2004 [6]. The INT type proved to be associated with considerably better prognosis than the PB subtype, which has been confirmed by several recent series $[3,4,7]$.

Our study aimed at a detailed analysis of clinical, pathological and immunohistochemical parameters for assessment of tumor biology and identification of prognostic factors after resection of periampullary adenocarcinomas of all four locations.

\section{Methods}

\section{Patients and data}

For the purpose of this study, periampullary adenocarcinomas were defined as pancreatic head PDAC, DBDAC, AMPAC or DUOAC. Only cases with resection by pancreatoduodenectomy, including conversion to total pancreatectomy due to positive intraoperative pancreatic resection margin were included. Thereby cases of the following WHO tumors [1] were excluded: PDAC not located in the pancreatic head, solid-pseudopapillary, acinar and neuroendocrine neoplasms, benign lesions, pancreatoblastoma, teratoma, mesenchymal tumors, lymphoma and secondary tumors. Patients operated at the Clinic for General and Visceral Surgery, University of Freiburg from 2001 to 2011 were identified and baseline and follow-up data extracted from a prospectively maintained database. All histopathological workup was performed at the Insitute of Pathology, University of Freiburg. Archived hematoxylin \& eosin (H\&E) stained slides were reevaluated by two experienced pathologists
(PB, IK) for accuracy of diagnosis and formalin-fixed and paraffin-embedded tissue (FFPE) blocks were selected for generation of serial tissue slices for immunohistochemistry (IHC). All cases with sufficient available FFPE for IHC were included in the study. The study protocol was approved by the Ethics Committee of the University of Freiburg (Ref 13/11).

\section{Standard pathological assessment}

During the study period (2001-2011), a standardized protocol was followed for diagnostic workup of pancreatoduodenectomy specimens: First, resection margins including the closest margin to the tumor and retroperitoneum towards portal vein and superior mesenteric artery were marked intraoperatively by the surgeon. After intraoperative transfer to the institute of pathology, every specimen was examined macroscopically by an experienced pathologist. Identifiable tumor masses or suspect areas were measured in three dimensions. Localization, size and distance of the tumor to the resection margins were documented and lymph node stations were separately evaluated. After formalin fixation and paraffin embedding, tissue slices of $3 \mu \mathrm{m}$ thickness were $\mathrm{H} \& \mathrm{E}$ stained. The following routine work up was equal in procedure for pylorus preserving pancreatoduodenectomy and the classical Whipple procedure. The number of routine tissue blocks and corresponding tissue slices was at minimum 10 (range 10 to 17 ). The standardized protocol comprised at least two samples for the enteral (oral and aboral) resection margins, and one sample for each of the following locations: whole circumferential parenchymal pancreas resection margin, tumor in relation to the closest posterior (retroperitoneum and vascular groove) resection margin, resection margin at the common bile duct, tumor in relation to the common bile duct and the main duct of the pancreas, tumor in relation to the duodenum, Papilla vateri. At minimum twelve regional lymph nodes were embedded in at least two routine tissue blocks. In case of portal venous en-bloc resections, one additional tissue sample in relation to the tumor was embedded. Additional tissue biopsies were embedded upon request of the operating surgeon. This standardized protocol was modified for total pancreatectomy as follows. The number of routine tissue blocks and corresponding tissue slices was minimal 11 (range 11 to 19). The resection margin of the splenic artery and vein and one sample of the spleen were embedded additionally, while the whole circumferential parenchymal pancreas resection margin was not embedded.

Histopathological reports included diagnosis according to WHO classification, UICC stage, presence or absence of lymphatic, vascular and perineural invasion and assessment of oral, aboral, biliary and posterior (retroperitoneal and vascular groove) resection margin. Additional 
immunohistochemistry for pancytokeratin was performed when it was felt necessary in difficult cases.

\section{Histologic workup}

For the present study, all cases were re-assessed by a surgeon (UFW) and two experienced pathologists (PB, $\mathrm{IK})$ in terms of clinical findings and history, preexisting macro- and histopathologic reports and H\&E stained tissue slides, as well as new $\mathrm{H} \& \mathrm{E}$ stained tissue slices to ensure correct diagnosis according to current WHO classification and anatomical tumor location. The site of the main tumor mass and/or eventual precursor lesions (intraductal papillary mucinous neoplasms of the pancreas (IPMN) or adenomatous lesions of duodenum, ampulla or bile duct) was used for definition. For this study, five histological subtypes were defined. Intestinal (INT), pancreatobiliary (PB) and mixed intestinalpancreatobiliary (MIX), as well as poorly differentiated (POOR) were defined according to Albores-Saavedra et al. [8]. An additional category was added for rare other phenotypes $(\mathrm{OTH})$, as mucinous and adenosquamous adenocarcinomas were found in our collective. According to Albores-Saavedra et al. [8], INT adenocarcinomas are characterized by well-formed tubular to elongate glands, complex cribriformed areas, and solid nests similar to colorectal adenocarcinoma, whereas PB adenocarcinomas show simple or branching glands and small solid nests of cells surrounded by abnormal desmoplastic stroma (Figure 1). Few tumors with both patterns equally distributed were assigned to the MIX type. Poorly differentiated carcinomas (POOR) are composed of solid sheets and nests admixed with densely packed, small irregular glands and individual cells with marked nuclear polymorphism, little or no mucin production and strong mitotic activity (Figure 1).

Tumors containing over $50 \%$ of stromal mucin were classified as mucinous, as proposed by the WHO classification [1] of ampullary tumors, while diagnosis of colloid carcinoma of the pancreas requires at least $80 \%$ of extracellular mucin pools combined with a characteristic well-differentiated cuboidal to columnar cellular morphology.

Histopathologic assessment was done by two independent pathologists blinded for the respective clinical outcome. For classification of histopathologic subtype and immunohistochemistry, the pathologists were blinded towards tumor location.

\section{Immunohistochemistry}

After individual case review, tissue slides of $3 \mu \mathrm{m}$ were prepared from representative FFPE blocks. Immunohistochemistry was carried out using commercially available ready-to-use antibodies for cytokeratin 7 (CK7, DAKO IR619), cytokeratin 20 (CK20, DAKO IR777), and caudal type homeobox 2 (CDX2, DAKO IR080), LINKER reagent (DAKO K8022) and EnVision ${ }^{\mathrm{TM}}$ Flex Visualization system (DAKO K8000) on an autostainer LINK 48 (DAKO, Hamburg, Germany) device according to the manufacturer's instructions. The specimens were counterstained with hematoxylin. Omission of primary antibodies served as negative controls and normal pancreatic and intestinal epithelia as internal positive control.

Representative images of immunohistochemical stains are shown in Figure 1. Immunostaining for CK7 and CK20 was considered positive when appropriate brown staining was seen in the tumor cell cytoplasm and immunostaining for CDX2 when appropriate brown staining was seen in the nucleus. CK7, CK20 and CDX2 expressions were established calculating the percentage of immunoreactive cells in the total number of tumor cells and rounding to decades. Only cases with more than $5 \%$ of positive tumor cells were regarded as positive.

\section{Statistics}

IBM SPSS Statistics Version 20 (SPSS Inc. Chicago, IL) was used for all statistical calculations. For exploratory and descriptive analysis, scale variables were expressed as median (range) and ordinal or dichotomous variables as absolute and relative frequencies. For assessment of diagnostic consistency, interrater reliability analysis using the Kappa statistic was performed. Survival data was plotted and analyzed according to the Kaplan-Meyer method. For univariate and multivariate statistical testing the following methods were used: Mann-Whitney test, Chi squared test, Spearman rank correlation, binary logistic regression, Logrank test and Cox regression. The significance level was set to $\mathrm{p}=0.05$ and all statistical tests were performed two-sided.

\section{Results}

\section{Clinico-pathological reassessment and baseline data}

From a total of 966 surgical patients assessed for eligibility (Figure 2), $n=462$ did not have periampullary adenocarcinomas, $\mathrm{n}=160$ had no tumor resection and in $n=143$ cases there was insufficient tissue left for the present study due to use for other studies. Reassessment of clinical and pathological reports as well as histopathologic reexamination of H\&E stained tissue slides in 201 cases resulted in correction of data entry errors and thus exclusion of two patients with neuroendocrine neoplasms and one patient with ovarian cancer metastasis to the periampullary region. Furthermore there was a change in diagnosis from PDAC to AMPAC in four patients and from AMPAC to PDAC in two patients. Follow-up survival data was complete due to regular follow-up within the Comprehensive Cancer Center Freiburg. Out of the 198 patients included in the study, 


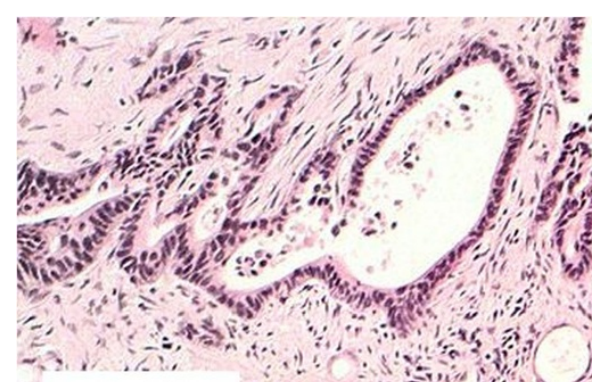

a) $\mathrm{HE}$

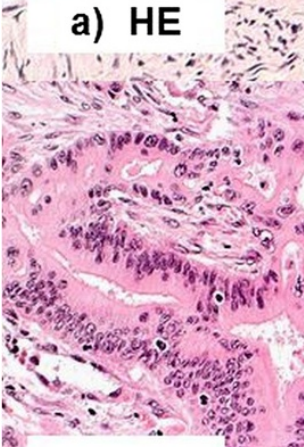

c) $\mathrm{HE}$

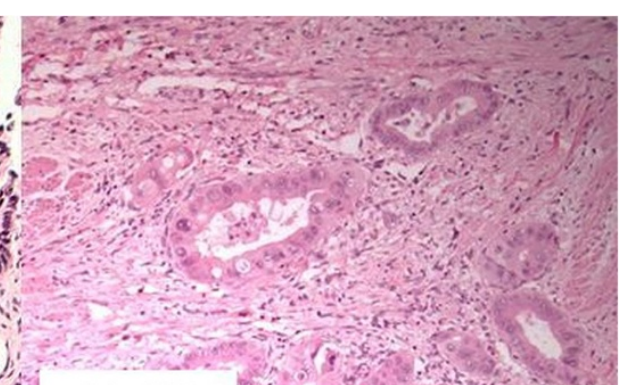

b) $\mathrm{HE}$

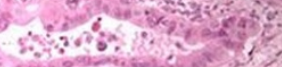

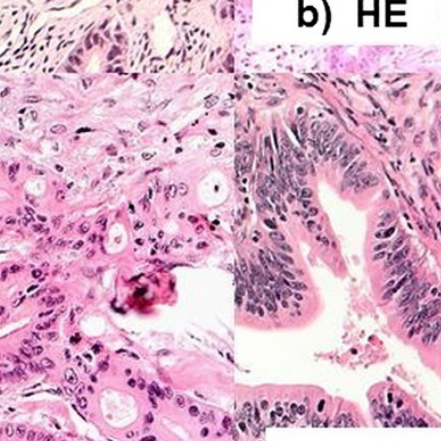

d) $\mathrm{HE}$

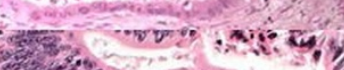
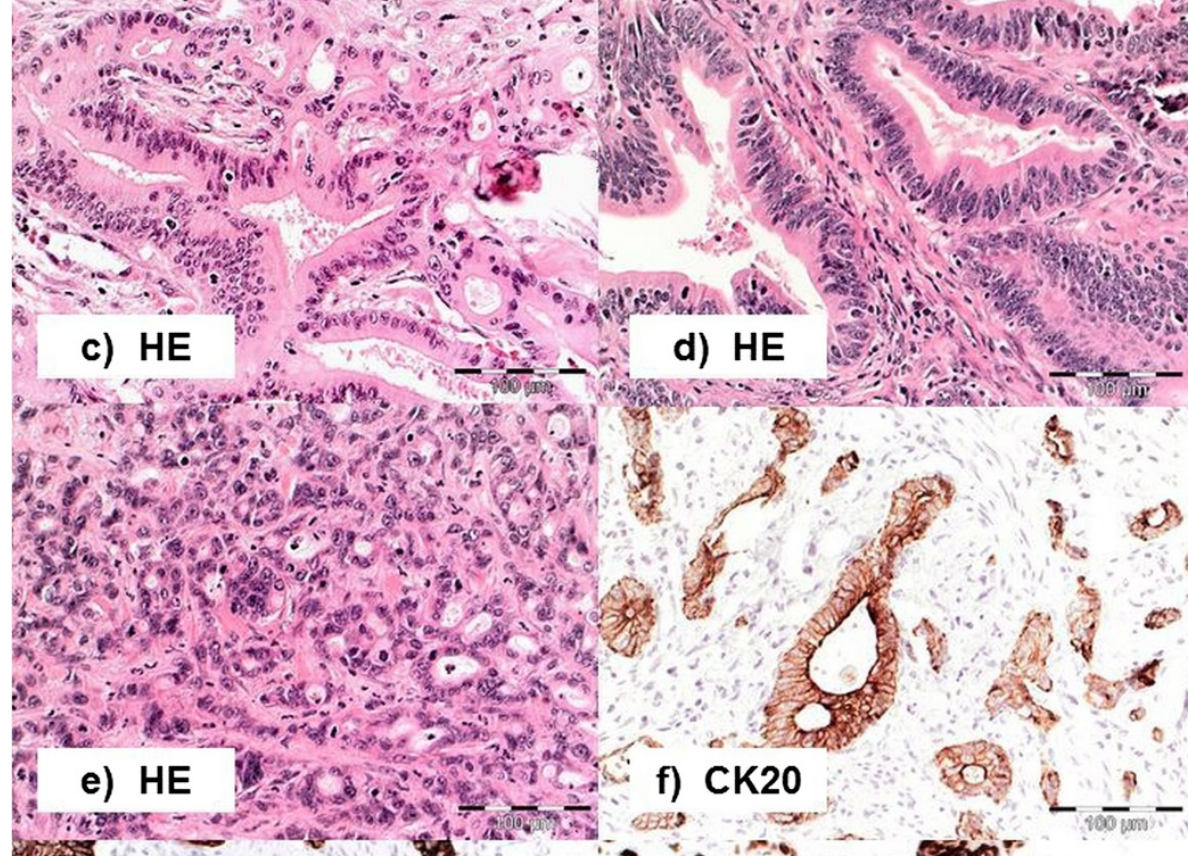

e) HE
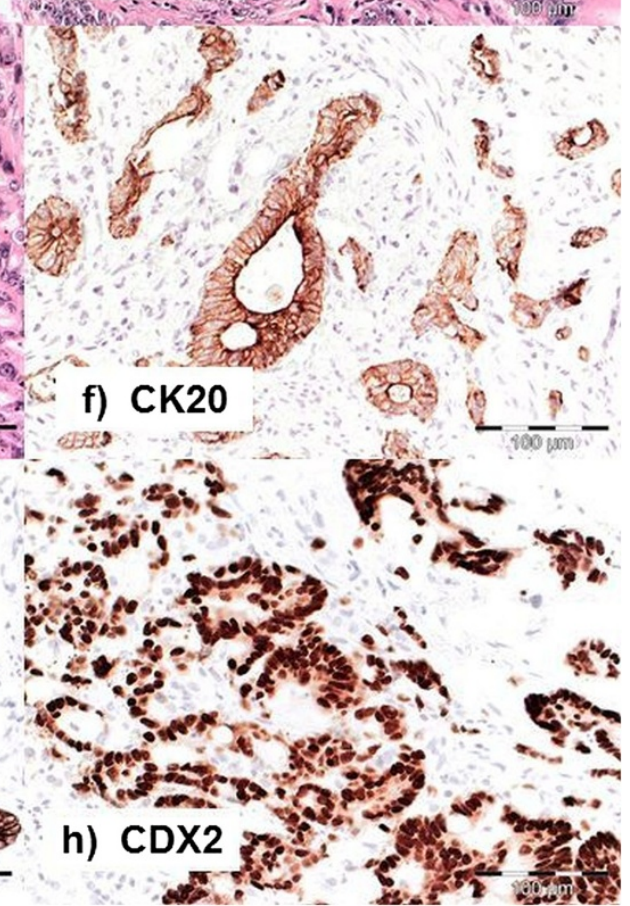

Figure 1 Histopathological Subtypes of Periampullary Adenocarcinomas. (a-e) hematoxylin-eosin stained tissue (H\&E): (a, b) adenocarcinoma of the pancreatobiliary type with simple branching glands lined by cuboidal columnar tumor cells with rounded nuclei and focally cribriform growth pattern. c adenocarcinoma of the mixed type showing both intestinal and pancreatobiliary growth pattern evenly distributed, $\mathbf{d}$ adenocarcinoma of the intestinal type with characteristic branching tubular glands, simple and pseudostratified mucin producing glandular epithelium with elongated hyperchromatic and pseudostratified nuclei, (e) poorly differentiated carcinoma displaying solid insular glandular growth pattern and remarkable nuclear atypia; (f-h) typical immunohistochemical staining patterns for cytokeratin 7 (CK7) in a pancreatobiliary type adenocarcinoma (g), cytokeratin 20 (CK20) (f) and caudal type homeobox 2 (CDX2) (h) in an intestinal type adenocarcinoma, pictures taken at 20-fold magnification. 


\section{assessed for eligibility}

patients operated for diseases of the pancreas, distal bile duct or duodenum 2001-2011 at the institution

$\mathrm{n}=966$

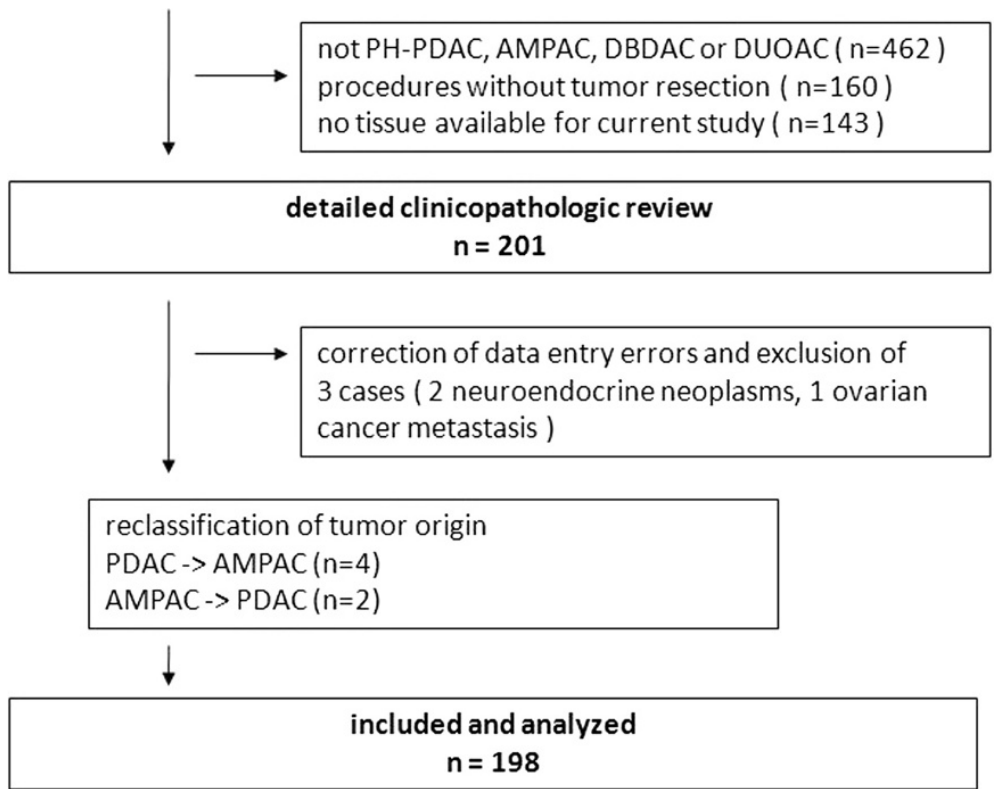

Figure 2 Flowchart of patient selection and data assessment. Abbreviations: PH-PDAC pancreatic head pancreatic ductal adenocarcinoma, DBDAC distal bile duct adenocarcinoma, AMPAC ampullary adenocarcinoma, DUOAC duodenal adenocarcinoma.

about two third had pancreatic cancer $(n=126)$. The second largest group was ampullary cancer $(n=40)$, followed by distal bile duct $(\mathrm{n}=23)$ and duodenal cancer ( $\mathrm{n}=9$ ) (Table 1).

Clinical and baseline data are shown in Table 1. No significant differences were found concerning age or gender distribution among the different tumor locations. 14 patients (7\%) had received neoadjuvant therapy before resection (13 PDAC and 1 AMPAC). Distant metastasis was very rare among resected patients (4\%). The usual resection procedure was pylorus-preserving pancreatoduodenectomy (PPPD, $n=163$ ), with few cases requiring extension to classical Whipple procedure (CWP, $\mathrm{n}=23$ ) or total pancreatectomy (TPE, $\mathrm{n}=12$ ) for tumor invasion of the pyloric region or intraoperative positive pancreatic resection margin, respectively. PDAC and DBDAC were significantly more often locally advanced as demonstrated by a higher rate of portal venous resections (PVR) due to adhesion to or infiltration of the portal vein or it's confluence (PDAC/DBDAC $42 \% / 26 \%$ vs $5 \% / 11 \%$ AMPAC/DUOAC). Perioperative mortality was $4 \%(n=7)$.

\section{Basic pathology and histological subtype analysis}

Results of explorative data analysis and statistical testing concerning basic pathology are depicted in Table 1.
Several statistically significant differences between the four groups could be noted. DUOAC had the largest median diameter $(40 \mathrm{~mm})$ while AMPAC $(20 \mathrm{~mm})$ and DBDAC $(15 \mathrm{~mm})$ were smaller and PDAC intermediate (27 mm). Only $53 \%$ of AMPAC were of stage T3 or T4, compared to $78-87 \%$ for the other entities. Likewise, only $8 \%$ of AMPAC had positive resection margins, compared to $22-33 \%$ for other locations. Adenomatous precursor lesions displayed statistically significant decreasing frequency from DUOAC/AMPAC (33\%/30\%) to DBDAC/PDAC (17\%/1\%). Nine percent of PDAC were associated with IPMN precursor lesions. High-grade intraepithelial dysplasia was present in 17 of 20 (85\%) associated adenomatous precursor lesions and in 9 of 11 (82\%) IPMN.

Results of histological subtype assessment are shown in Table 1. The distribution of subtypes was significantly different among the adenocarcinoma groups. The INT type adenocarcinoma showed decreasing frequency with distance from the duodenum in the order DUOAC AMPAC - DBDAC - PDAC, ranging from 56\% to $9 \%$, while the percentage of $\mathrm{PB}$ type increased from $22 \%$ to $71 \%$. Of note, two DUOAC were assigned to the PB phenotype by application of the subtype criteria. MIX and OTH types were not frequently encountered ( 0 - 9\% and $0-11 \%)$. The percentage of poorly differentiated 
Table 1 Baseline parameters, basic pathology and immunohistochemical markers for 198 patients with periampullary adenocarcinomas

\begin{tabular}{|c|c|c|c|c|c|c|c|}
\hline \multirow{2}{*}{\multicolumn{2}{|c|}{$\begin{array}{c}\text { Parameter } \\
\text { N }\end{array}$}} & Total & PDAC & DBDAC & AMPAC & DUOAC & $\mathrm{p}$ \\
\hline & & 198 & 126 & 23 & 40 & 9 & - \\
\hline \multicolumn{2}{|c|}{ Male : female } & 102:96 & $63: 63$ & $14: 9$ & $22: 18$ & $3: 6$ & 0.511 \\
\hline \multicolumn{2}{|l|}{ Age } & $67(30-89)$ & $67(30-89)$ & $69(49-83)$ & $65(36-84)$ & $66(46-78)$ & 0.137 \\
\hline \multicolumn{2}{|c|}{ Distant metastasis (M1) } & $7 / 4 \%$ & $4 / 3 \%$ & $0 / 0 \%$ & $2 / 5 \%$ & $1 / 11 \%$ & 0.448 \\
\hline \multicolumn{2}{|c|}{ Neoadjuvant therapy } & $14 / 7 \%$ & $13 / 10 \%$ & $0 / 0 \%$ & $1 / 3 \%$ & $0 / 0 \%$ & 0.126 \\
\hline \multirow[t]{4}{*}{ Resection } & PPPD & 163 & 103 & 19 & 34 & 7 & 0.803 \\
\hline & Whipple & 23 & 14 & 2 & 5 & 2 & \\
\hline & total PE & 12 & 9 & 2 & 1 & 0 & \\
\hline & PVR & $62 / 31 \%$ & $53 / 42 \%$ & $6 / 26 \%$ & $2 / 5 \%$ & $1 / 11 \%$ & 0.000 \\
\hline \multicolumn{2}{|c|}{ Perioperative mortality } & $7 / 4 \%$ & $5 / 4 \%$ & $2 / 9 \%$ & $0 / 0 \%$ & $0 / 0 \%$ & 0.300 \\
\hline \multicolumn{2}{|c|}{ Tumor size $(\mathrm{mm})$} & $25(1-320)$ & $27(1-80)$ & $15(8-25)$ & $20(2-320)$ & $40(22-60)$ & 0.000 \\
\hline \multicolumn{2}{|c|}{ Grade G3/4 } & $72 / 37 \%$ & $48 / 39 \%$ & $8 / 35 \%$ & $12 / 31 \%$ & $4 / 44 \%$ & 0.785 \\
\hline \multicolumn{2}{|c|}{ Stage $T 3 / 4$} & $155 / 78 \%$ & $109 / 87 \%$ & $18 / 78 \%$ & $21 / 53 \%$ & $7 / 78 \%$ & 0.000 \\
\hline \multicolumn{2}{|c|}{ Stage N1/2 } & 132 / 67\% & $89 / 71 \%$ & $14 / 61 \%$ & $24 / 60 \%$ & $5 / 56 \%$ & 0.468 \\
\hline \multicolumn{2}{|c|}{ Lymph node ratio (LNR) } & $.09(0-.86)$ & $.10(0-.86)$ & $.08(0-.72)$ & $.07(0-.71)$ & $.10(0-.28)$ & 0.694 \\
\hline \multicolumn{2}{|c|}{ Lymphangiosis (L1) } & $87 / 44 \%$ & $60 / 48 \%$ & $5 / 22 \%$ & $19 / 48 \%$ & $3 / 33 \%$ & 0.116 \\
\hline \multicolumn{2}{|c|}{ Hemangiosis (V1) } & $29 / 15 \%$ & $22 / 18 \%$ & $3 / 13 \%$ & $2 / 5 \%$ & $2 / 22 \%$ & 0.237 \\
\hline \multicolumn{2}{|c|}{ Perineural invasion (Pn1) } & $113 / 57 \%$ & $88 / 70 \%$ & $12 / 52 \%$ & $13 / 33 \%$ & $0 / 0 \%$ & 0.000 \\
\hline \multirow[t]{2}{*}{ Associated precursor } & Adenoma & $20 / 10 \%$ & $1 / 1 \%$ & $4 / 17 \%$ & $12 / 30 \%$ & $3 / 33 \%$ & 0.000 \\
\hline & IPMN & $11 / 6 \%$ & $11 / 9 \%$ & $0 / 0 \%$ & $0 / 0 \%$ & $0 / 0 \%$ & 0.084 \\
\hline \multicolumn{2}{|c|}{ Positive resection margin } & $51 / 26 \%$ & $41 / 33 \%$ & $5 / 22 \%$ & $3 / 8 \%$ & $2 / 22 \%$ & 0.017 \\
\hline \multicolumn{8}{|c|}{ Subtype } \\
\hline \multicolumn{2}{|l|}{ INT } & $39 / 20 \%$ & $11 / 9 \%$ & $5 / 22 \%$ & $18 / 45 \%$ & $5 / 56 \%$ & 0.000 \\
\hline \multicolumn{2}{|l|}{ MIX } & $12 / 6 \%$ & $8 / 6 \%$ & $2 / 9 \%$ & $2 / 5 \%$ & $0 / 0 \%$ & \\
\hline \multicolumn{2}{|l|}{ PB } & $118 / 16 \%$ & $89 / 71 \%$ & $12 / 52 \%$ & $15 / 38 \%$ & $2 / 22 \%$ & \\
\hline \multicolumn{2}{|l|}{$\mathrm{OTH}$} & $6 / 3 \%$ & $5 / 4 \%$ & $0 / 0 \%$ & $0 / 0 \%$ & $1 / 11 \%$ & \\
\hline \multicolumn{2}{|c|}{ POOR } & $23 / 12 \%$ & $13 / 10 \%$ & $4 / 17 \%$ & $5 / 13 \%$ & $1 / 11 \%$ & \\
\hline \multicolumn{8}{|c|}{ Immunohistochemical Markers } \\
\hline \multicolumn{2}{|c|}{$\%$ CK7+ } & $90(0-100)$ & $90(0-100)$ & $95(0-100)$ & $80(0-100)$ & $0(0-60)$ & 0.000 \\
\hline \multicolumn{2}{|c|}{$\%$ CK20+ } & $0(0-100)$ & $0(0-100)$ & $0(0-100)$ & $10(0-100)$ & $70(10-100)$ & 0.000 \\
\hline \multicolumn{2}{|c|}{$\%$ nuclear CDX2+ } & $10(0-100)$ & $0(0-100)$ & $0(0-100)$ & $50(0-100)$ & $95(60-100)$ & 0.000 \\
\hline
\end{tabular}

Values are depicted as absolute and percentage of column for categorial and median (range) for scale variables if not otherwise specified. $\mathrm{p}$ value derived from two-sided Median test (scale variables), Chi squared test (categorial variables) and from two-sided Spearman rank correlation test (immunohistochemical markers), each for the respective rows.

Abbreviations: PPPD pylorus preserving pancreatoduodenectomy, Whipple classical Whipple procedure, $P E$ pancreatectomy, $P V R$ portal venous resection. INT intestinal, $P B$ pancreatobiliary, MIX mixed intestinal-pancreatobiliary, POOR poorly differentiated carcinoma, OTH other specific subtypes, + positive, $C K$ cytokeratin, $P D A C$ pancreatic ductal adenocarcinoma, DBDAC distal bile duct adenocarcinoma, AMPAC ampullary adenocarcinoma, DUOAC duodenal adenocarcinoma.

carcinomas was relatively homogenous ranging from 10 to $17 \%$. Rare WHO subtypes designated as OTH were mucinous (2 PDAC and 1 DUOAC) and adenosquamous (3 PDAC). Interrater reliability concerning the five defined histological subtypes was found to be very good (Kappa $=0.920, \mathrm{p}=0.000)$.

\section{Diagnostic value of immunohistochemical markers}

Results of immunohistochemical marker assessment are presented in Table 1. Cytokeratin 7 (CK7) is a marker of pancreatobiliary epithelia, whereas cytokeratin
20 (CK20) and CDX2 are expressed by intestinal epithelia. Median intestinal marker expression was highest in DUOAC (CK20 and CDX2, 70\% and 95\%) and decreased for AMPAC (10\% and 50\%) and negative for DBDAC and PDAC (median expression level 0\%). The pancreatobiliary marker CK7 showed the inverse pattern with high median expression level in PDAC / DBDAC (90\%/ 95\%), decreased with AMPAC (85\%) and $0 \%$ in DUOAC. These correlations were highly significant $(\mathrm{p}<0.001$ in two-sided Spearman rank correlation test). 
Uni- and multivariate binary logistic regression models were used in the attempt to predict the INT type by means of the immunohistochemical markers CK7, CK20 and CDX2 (details shown in Table 2). However, due to poor performance for prediction of the INT type in PDAC / DBDAC location (0\%-13\% correct prediction for univariate and $19 \%$ for multivariate model), even a multivariate model including all three markers only achieved 51\% correct prediction for the INT type when applied to the total patient collective.

\section{Baseline data and biology of the intestinal subtype}

Results of a subgroup analysis of INT adenocarcinomas are shown in Table 3. While median tumor size did not differ between INT and NON-INT type (23 vs $25 \mathrm{~mm}$, $\mathrm{p}=0.779$ ), several attributes of lower malignant potential could be demonstrated for INT adenocarcinomas. High tumor grade, high $\mathrm{T}$ and $\mathrm{N}$ Stage, lymph node ratio (LNR), perineural and vascular invasion were significantly less frequent and expression of intestinal molecular markers CK20 and CDX2 was significantly higher with INT versus non-INT adenocarcinoma. INT adenocarcinomas were more frequently located in the ampulla of Vater or duodenum region than in the pancreas or distal bile duct and margin-free surgical resection was more frequently achieved. There was a statistically significant association of the INT type with adenomatous precursor lesions, but not with IPMN precursor lesions.
Survival analysis for tumor location and histological subtype Details of survival analysis regarding tumor location and subtype are shown in Table 4 and Figure 3. Perioperative deaths $(n=7)$ were excluded from survival analysis. Overall median follow-up was 15 months (range 0116 months). Median survival ranged in ascending order from 23, 29, 64 to 71 months for PDAC, DBDAC, AMPAC to DUOAC. For histological subtypes, median survival was 13, 22, 25 and 30 months for POOR, PB, OTH and MIX subtype, while median survival was not yet reached for the INT subtype. Pairwise comparison by two-sided logrank test among histological subtypes disclosed that significant differences existed only between INT type versus other types. Therefore, nonintestinal types (NON-INT) were assigned to a common group. When survival plots were stratified by tumor location, survival after resection of INT type adenocarcinomas was remarkably favorable for each location, reaching the significance level for PDAC, AMPAC and DUOAC (Table 4 and Figure 3).

\section{Univariate and multivariate survival analysis}

Results of uni- and multivariate survival analysis are shown in Table 5 . Cases with perioperative death $(n=7)$ were excluded from survival analysis. In univariate analysis, several clinicopathologic parameters were found to be significantly associated with poor survival: necessity of portal venous resection, high tumor grade, $\mathrm{T}$ stage, $\mathrm{N}$ stage and LNR, lymphatic and blood vessel invasionas well as positive resection margins. Presence of a precursor lesion was significantly associated with

Table 2 Diagnostic value of immunohistochemical markers for intestinal type adenocarcinoma

\begin{tabular}{|c|c|c|c|c|c|c|}
\hline \multirow[t]{3}{*}{ Location } & \multirow[t]{3}{*}{ Marker } & \multirow{2}{*}{\multicolumn{2}{|c|}{$\begin{array}{c}\% \text { Positive tumor cells } \\
\text { Median (Range) }\end{array}$}} & \multicolumn{2}{|c|}{$\%$ Correct prediction } & \multirow[t]{3}{*}{$p$} \\
\hline & & & & \multirow[b]{2}{*}{ INT } & \multirow[b]{2}{*}{ NON-INT } & \\
\hline & & INT & NON-INT & & & \\
\hline \multirow[t]{5}{*}{ PDAC / DBDAC } & CK7 & $90(0-100)$ & $90(0-100)$ & $0 \%$ & $100 \%$ & 0.288 \\
\hline & CK20 & $10(0-100)$ & $0(0-100)$ & $0 \%$ & $100 \%$ & 0.034 \\
\hline & CDX2 & $45(0-100)$ & $0(0-90)$ & $13 \%$ & $100 \%$ & 0.000 \\
\hline & CK7, CK20, CDX2 & & & $19 \%$ & $100 \%$ & 0.002 \\
\hline & multivariate & & & & & \\
\hline \multirow[t]{5}{*}{ AMPAC / DUOAC } & CK7 & $20(0-100)$ & $85(0-100)$ & $57 \%$ & $75 \%$ & 0.024 \\
\hline & CK20 & $80(0-100)$ & $8(0-100)$ & $65 \%$ & $79 \%$ & 0.002 \\
\hline & $\mathrm{CDX} 2$ & $90(20-100)$ & $30(0-100)$ & $87 \%$ & $79 \%$ & 0.000 \\
\hline & CK7, CK20, CDX2 & & & $87 \%$ & $75 \%$ & 0.000 \\
\hline & multivariate & & & & & \\
\hline \multirow[t]{2}{*}{ ALL } & CK7, CK20, CDX2 & & & $51 \%$ & $95 \%$ & 0.000 \\
\hline & multivariate & & & & & \\
\hline
\end{tabular}

Prediction modeled by univariate and multivariate binary logistic regression analysis, $\mathrm{p}$ values given for the two-sided overall model omnibus test of the respective row.

Abbreviations: PDAC / DBDAC / AMPAC / DUOAC pancreatic ductal/distal bile duct / ampullary / duodenal adenocarcinoma, INT intestinal, NON-INT non-intestinal, $C K$ cytokeratin, $\mathrm{Cl}$ confidence interval. 
Table 3 Baseline data and biology of the intestinal subtype

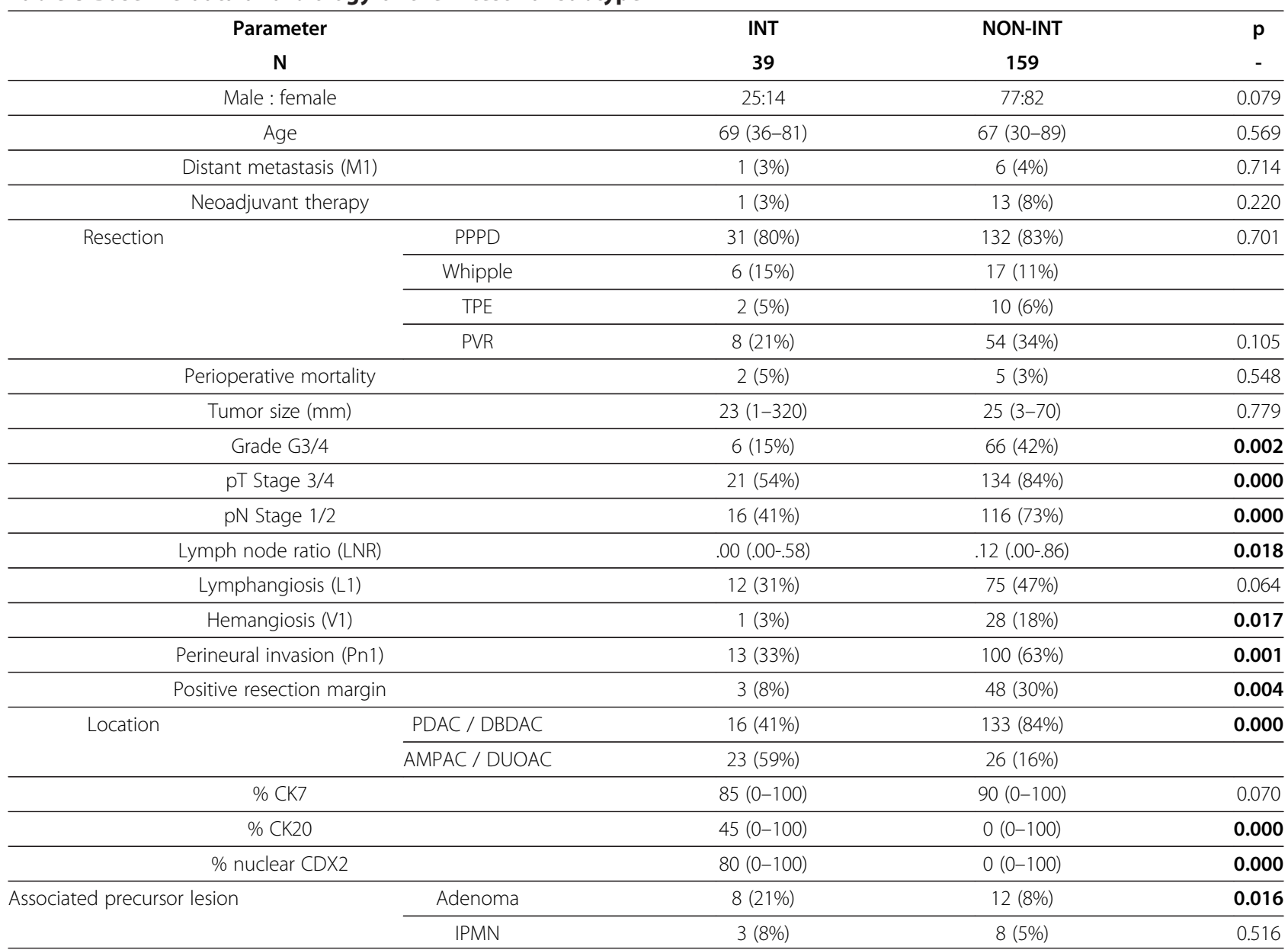

Values are depicted as absolute and percentage of column for categorial and median (range) for scale variables, $\mathrm{p}$ value derived from Mann-Whitney (scale variables) or Chi squared test (categorial variables) for the respective row.

Abbreviations: Whipple / PPPD non / pylorus-preserving pancreatoduodenectomy, TPE total pancreatectomy, PVR portal venous resection, PDAC/DBDAC/AMPAC/ DUOAC pancreatic ductal/distal bile duct/ampullary/duodenal adenocarcinoma, NON-/INT non-/intestinal subtype, CK cytokeratin.

better survival. This was however only the cause for adenomatous precursor lesions in non-pancreatic adenocarcinomas ( $\mathrm{p}=0.017$, two-sided Logrank test), as the subgroup of PDAC with associated IPMN did not show significantly better survival than PDAC without associated IPMN ( $\mathrm{p}=0.538$, Logrank test). Both tumor location and histological subtype were highly significant predictors of survival $(\mathrm{p}<0.001)$. Accordingly, high CK7 expression and low CDX2 expression, as characteristic of NON-INT phenotype, were significant predictors of poor survival.

Parameters found to be significant in univariate analysis were included in a Cox proportional hazards model including all cases with periampullary adenocarcinomas $(n=191$ after exclusion of $n=7$ cases of perioperative mortality). In this model, only histological subtype (Hazard Ratio 1.5, $\mathrm{p}=0.006$ ) and LNR (Hazard Ratio 2.3, p =0.019) were found to represent independent predictors of survival. This was confirmed by conditional backward elimination of predictive parameters.

\section{Discussion}

Only one group has so far systematically extended the INT/PB concept to the whole spectrum of periampullary adenocarcinomas $[3,9]$. We were able to validate and extend these findings in our study. From a plethora of prognostic markers identified in univariate analysis, including UICC stage, tumor size, resection margin status and even immunohistochemical markers, only histologic subtype and lymph node ratio qualified as independent predictors of survival. Hereby the present study confirms that histopathological differentiation, in contrast to tumor location, is an independent prognostic factor. It also confirms that the lymph node ratio is a stronger independent prognostic marker than $\mathrm{N}$ stage, 
Table 4 Univariate survival analysis for tumor location and histopathological subtypes in periampullary adenocarcinomas

\begin{tabular}{|c|c|c|c|c|c|c|}
\hline \multirow[t]{2}{*}{ Location } & \multirow[t]{2}{*}{ Type } & \multirow[t]{2}{*}{$\mathrm{N}$} & \multirow{2}{*}{$\begin{array}{l}\text { Events } \\
\text { (Deaths) }\end{array}$} & \multicolumn{2}{|c|}{ Survival (months) } & \multirow[t]{2}{*}{$\mathrm{p}$} \\
\hline & & & & Median & Mean & \\
\hline & & \multicolumn{5}{|c|}{ Comparison of tumor locations versus PDAC } \\
\hline PDAC & ALL & 121 & 64 & 23 & 31 & - \\
\hline DBDAC & & 21 & 14 & 29 & 39 & 0.430 \\
\hline AMPAC & & 40 & 14 & 64 & 72 & 0.000 \\
\hline \multirow[t]{2}{*}{ DUOAC } & & 9 & 6 & 71 & 46 & 0.169 \\
\hline & & \multicolumn{5}{|c|}{ Comparison of tumor subtypes versus INT } \\
\hline \multirow[t]{7}{*}{ ALL } & INT & 37 & 8 & NR & 83 & - \\
\hline & MIX & 12 & 8 & 30 & 37 & 0.002 \\
\hline & $\mathrm{OTH} \mathrm{WHO}$ & 6 & 4 & 25 & 38 & 0.017 \\
\hline & PB & 115 & 66 & 22 & 34 & 0.000 \\
\hline & POOR & 21 & 12 & 13 & 28 & 0.000 \\
\hline & NON-INT & 154 & 90 & 22 & 35 & 0.000 \\
\hline & & \multicolumn{5}{|c|}{$\begin{array}{l}\text { Comparison INT vs NON-INT stratified } \\
\text { for tumor location }\end{array}$} \\
\hline \multirow[t]{2}{*}{ PDAC } & NON-INT & 111 & 63 & 20 & 29 & 0.034 \\
\hline & INT & 10 & 1 & 39 & 74 & \\
\hline \multirow[t]{2}{*}{ DBDAC } & NON-INT & 17 & 12 & 12 & 37 & 0.563 \\
\hline & INT & 4 & 2 & 29 & 55 & \\
\hline \multirow[t]{2}{*}{ AMPAC } & NON-INT & 22 & 11 & 38 & 56 & 0.019 \\
\hline & INT & 18 & 3 & NR & 90 & \\
\hline \multirow[t]{2}{*}{ DUOAC } & NON-INT & 4 & 4 & 4 & 10 & 0.003 \\
\hline & INT & 5 & 2 & 75 & 75 & \\
\hline
\end{tabular}

Survival estimates are derived from Kaplan-Meier method, $p$ values from twosided Logrank test. For Kaplan-Meier plots see Figure 3. Perioperative deaths $(n=7)$ were excluded from survival analysis.

Abbreviations: INT intestinal, NON-INT non-intestinal, $P B$ pancreatobiliary subtype, MIX mixed subtype, $P O O R$ poorly differentiated carcinoma, OTH other subtypes, $P D A C$ pancreatic ductal adenocarcinoma, DBDAC distal bile duct adenocarcinoma, AMPAC ampullary adenocarcinoma, DUOAC duodenal adenocarcinoma, $N R$ not reached.

which has been shown for PDAC, AMPAC and DBDAC before $[10,11]$.

We were able to demonstrate a significantly better survival of the INT subtype not only for AMPAC but also in the subgroups of DUOAC and PDAC, which has not been demonstrated before due to insufficient case numbers $[3,9]$. Probably due to its relatively rare occurrence (around 10\% of PDAC), INT type PDAC has only recently been recognized [12].

The concept of INT and PB differentiation has recently been extended to IPMN. It has been suggested that INT type IPMN can develop into colloid carcinoma (CAC) $[13,14]$, which is associated with better survival $[15,16]$. We show that in ampullary and duodenal location, INT phenotype is associated with adenomatous precursor lesions, but this is not the case in PDAC, validating its existence as a separate entity apart from colloid carcinoma or invasive IPMN. In addition, one recent study shows no association of intraductal papillary neoplasms of the bile duct (IPNB) with INT type cholangiocarcinoma $[17,18]$. It may therefore be suggested that an "intestinal pathway" of carcinogenesis is possible via the INT type of IPMN to colloid carcinoma, and duodenal or ampullary adenoma to DUOAC and AMPAC, but also without papillary precursor lesions to INT type PDAC and DBDAC.

On the other hand, blinded application of the INT/PB classification scheme resulted in assignment of two DUOAC to the PB group. One might speculate that if INT type adenocarcinoma can arise in the pancreas, vice versa $\mathrm{PB}$ type adenocarcinoma may arise in rare instances in the duodenum due to common embryologic origin, but further evaluation is currently not possible given the exceeding rarity of DUOAC.

As CK7, CK20 and CDX2 are known to be fairly specific markers for the discrimination of INT and PB subtypes in AMPAC and IPMN [14,19-22], it was surprising that this was not the case for PDAC and DBDAC in our study. This finding is noteworthy as it shows that careful morphological assessment by an experienced pathologist outperforms immunohistochemical markers in this respect.

This study has several limitations that have to be mentioned. First, although data was obtained from a prospectively maintained database, all data was analyzed retrospectively. There was a relatively high number of cases assessed for eligibility from which insufficient amounts of tissue were available for the present study due to use for other studies. A selection bias by exclusion of small tumors with few tissue can thus not totally be ruled out. Furthermore we note a relatively high rate of PDAC in our series compared to other recent studies [2,4] on this subject. This may be explained in several ways: first, selection bias excluding small tumors may favor the inclusion of PDAC which are usually larger than AMPAC; furthermore assignment to tumor location was not performed according to the most recent detailed protocols $[2,4]$ and could only be reassessed in a retrospective manner. Misclassification of origin especially in large tumors cannot totally be ruled out. Nevertheless pathologists and surgeons at our institution since long have been aware of this complex issue and extensive surgical and pathological experience exists. We believe that by primary assessment in a standardized protocol as well as detailed re-assessment in the scope of this study, risk of misclassification was reduced to a minimum.

Another current issue concerns resection margin status in PDAC. Some recent studies demonstrated by extensive resection margin workup that the majority of 


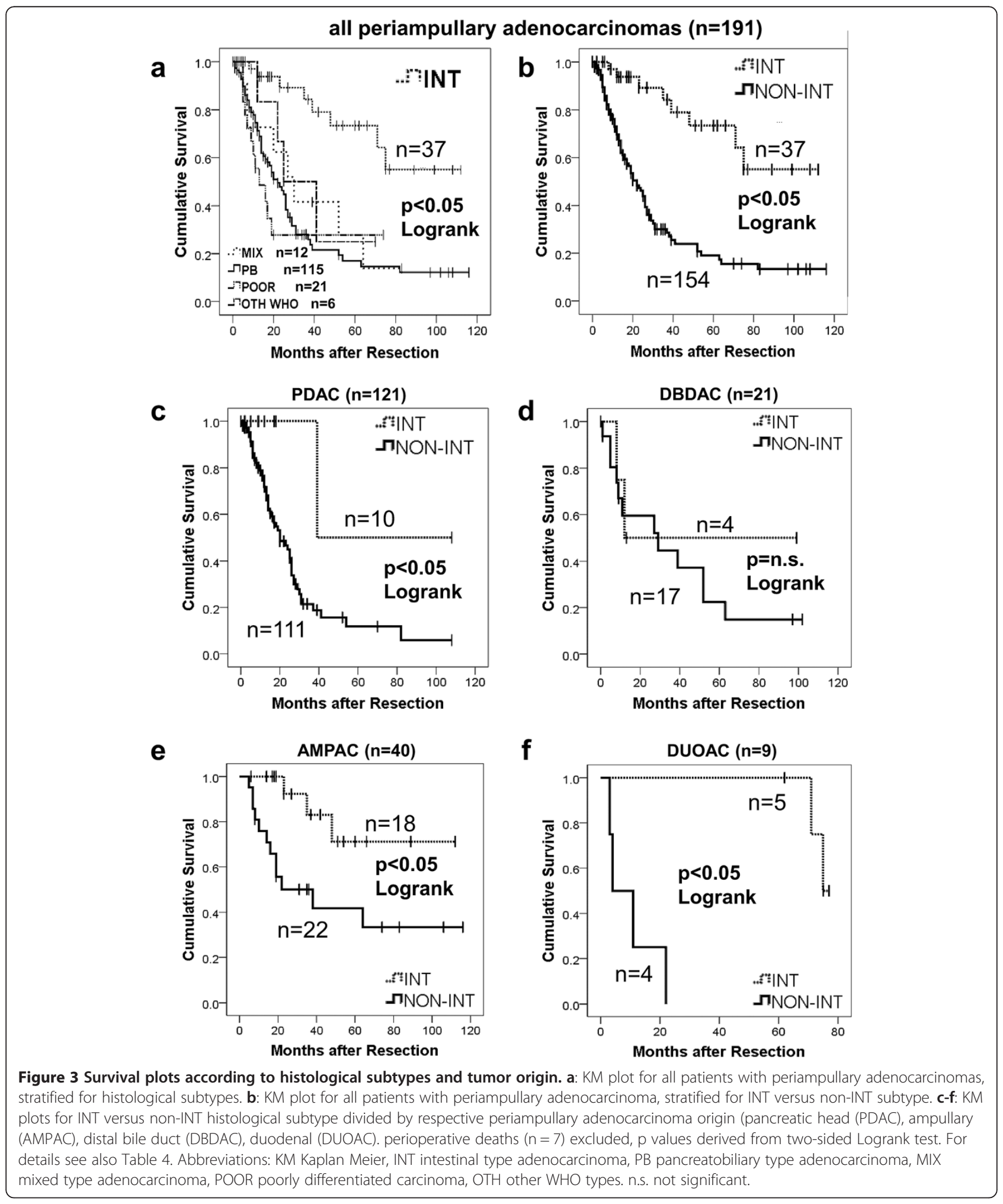

PDAC resections were in fact margin positive (R1) [23-25]. Margin assessment in our standardized protocol was very detailed but not as extensive as in some of the aforementioned studies, thus a number of R0 might in fact have been $\mathrm{R} 1$ resections. Nevertheless our data is validated by the fact that margin status was a prognostic 
Table 5 Univariate and multivariate survival analysis for resected periampullary adenocarcinomas

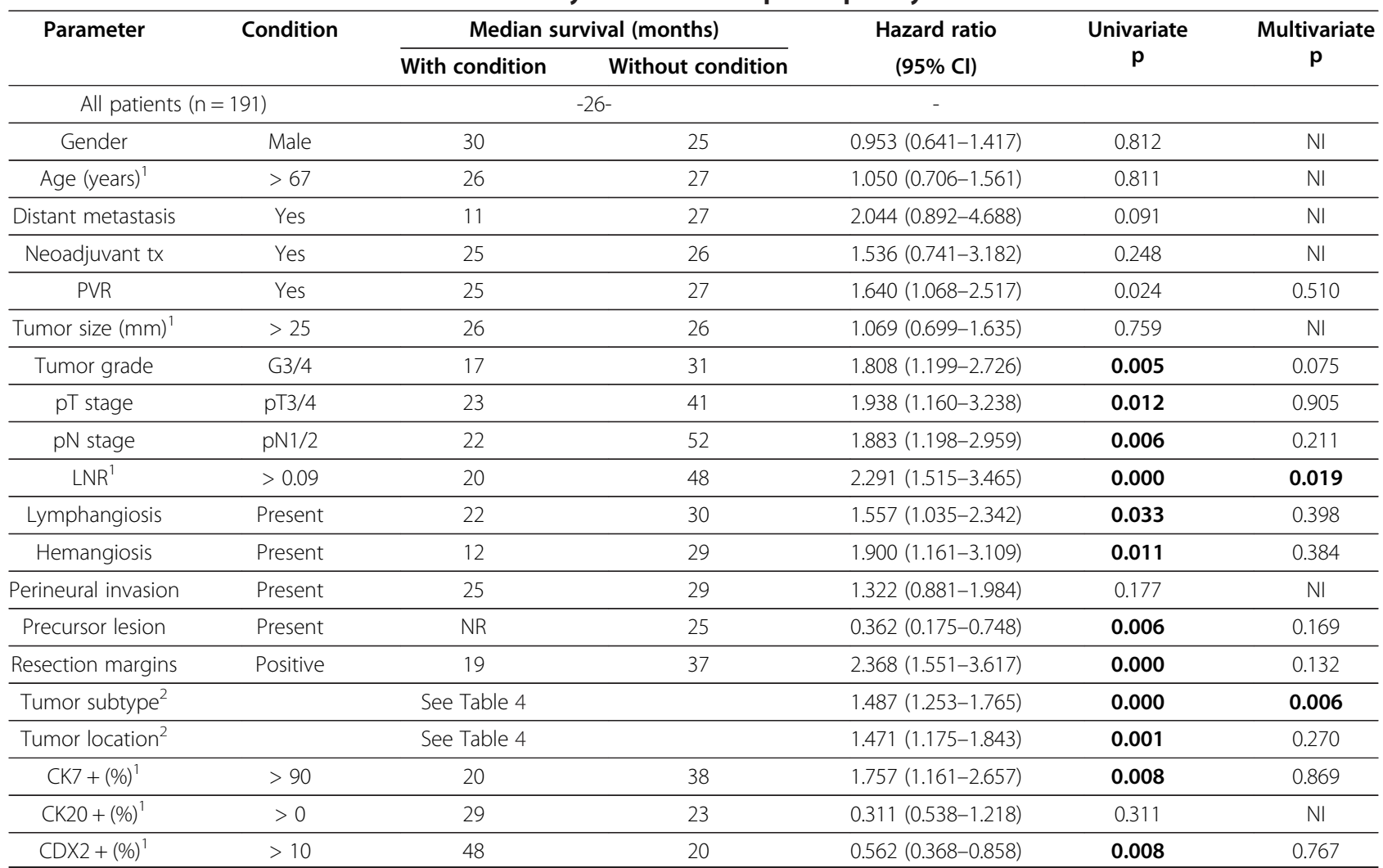

Survival estimates are derived from Kaplan-Meyer method, perioperative deaths $(n=7)$ were excluded, univariate / multivariate $p$ is derived from two-sided Cox regression in a proportional hazards model.

Abbreviations: $C K$ cytokeratin, $C l$ confidence interval, + positive. ${ }^{1}$ cut-off at overall median value, ${ }^{2}$ defined as ordinal variable according to decreasing median survival as indicated in Table 2, pos positive, tx therapy, PVR portal venous resection, NR not reached.

factor. More detailed analysis of this issue was not within the scope of this study but will have to be addressed in future studies.

The most important aspect of pathological diagnostic is clinical decision making on the basis of prognostic factors. With respect to the findings of this study, several aspects warrant consideration.

Based on favorable survival, surgical treatment of metastasis may be indicated in INT type AMPAC, as already suggested by others [26]. Given the fact that tumor location is less relevant than subtype, this concept may be extended to PDAC, DBDAC and DUOAC. Especially since criteria for the rarely performed resection of metastatic PDAC are poorly defined [27], subtype might be a valuable adjunct in decision making.

Furthermore, there is still no consensus regarding the indication and regimen for adjuvant therapy in nonpancreatic periampullary adenocarcinomas [28-33]. In clinical practice, treatment regimen for AMPAC, DUOAC or DBDAC are usually extrapolated from PDAC. Limited data suggests that patients with node positive and margin-negative AMPAC benefit from adjuvant therapy $[28,30,33]$. However, these studies did not report on subtype. Regarding the results of our study, a more differentiated approach based on subtype may be suggested for future trials.

It has been shown that survival figures of series reporting outcome of resected pancreatic head cancer have been biased by inclusion of ampullary cancers [23,34]. In consequence, more thorough examination of tumor origin has been demanded by some authors [2]. However given the results of our study, the biologically valid and logistically preferable approach would be to distinguish between INT and $\mathrm{PB}$ differentiation rather than tumor location. This is emphasized by the fact that for larger tumors with involvement of all periampullary structures, assessment of primary location is very difficult or impossible.

\section{Conclusion}

In summary, our study demonstrates that histopathological subtype and lymph node ratio are the most important prognostic factors in periampullary adenocarcinomas, outperforming all other parameters. As tumor location becomes irrelevant with correct microscopical phenotype classification, this may obviate the need for time-intensive macroscopical and microscopical workup of tumor 
location in pancreatoduodenectomy specimen. It must be recommended that studies and trials on outcome and therapy of periampullary adenocarcinomas stratify for intestinal and pancreatobiliary differentiation.

\section{Competing interests}

The authors have no competing interests to declare.

\section{Authors' contributions}

$\mathrm{PB}$, IK and MW performed the pathological assessment and critically revised the manuscript. DB, SK and JH collected clinical data and performed the operations. TK and UTH performed the operations and critically revised the manuscript. UFW and FM collected the data, performed statistical analysis and drafted the manuscript. All authors read and approved the final manuscript.

\section{Acknowledgements}

This study was funded by a research grant of the German Research Foundation to Ulrich Friedrich Wellner (grant number DFG WE 5085/1-1).

\section{Author details}

'Institute of Pathology, University of Freiburg, Breisacher Str. 115a, Freiburg 79106, Germany. ${ }^{2}$ Clinic for General and Visceral Surgery, University of Freiburg, Hugstetter Str. 55, Freiburg 79106, Germany. ${ }^{3}$ Clinic for Surgery, University of Schleswig-Holstein Campus Lübeck, Ratzeburger Allee 160, Lübeck 23582, Germany.

Received: 20 March 2013 Accepted: 17 September 2013 Published: 22 September 2013

\section{References}

1. Bosman FT, Carneiro F, Hruban RH, Theise ND: WHO classification of tumors of the digestive system. 4th edition. Lyon: International Agency for Research in Cancer; 2010.

2. Adsay V, Ohike N, Tajiri T, Kim GE, Krasinskas A, Balci S, Bagci P, Basturk O, Bandyopadhyay S, Jang K-T, Kooby DA, Maithel SK, Sarmiento J, Staley CA, Gonzalez RS, Kong SY, Goodman M: Ampullary region carcinomas: definition and site specific classification with delineation of four clinicopathologically and prognostically distinct subsets in an analysis of 249 cases. Pathol: Am J Surg; 2012

3. Westgaard A, Tafjord S, Farstad IN, Cvancarova M, Eide TJ, Mathisen O, Clausen OPF, Gladhaug IP: Pancreatobiliary versus intestinal histologic type of differentiation is an independent prognostic factor in resected periampullary adenocarcinoma. BMC Cancer 2008, 8:170.

4. Pomianowska E, Grzyb K, Westgaard A, Clausen OPF, Gladhaug IP: Reclassification of tumour origin in resected periampullary adenocarcinomas reveals underestimation of distal bile duct cancer. Eur I Surg Oncol 2012, 38:1043.

5. Ohike N, Kim GE, Tajiri T, Krasinskas A, Basturk O, Coban I, Bandyopadhyay S, Morohoshi T, Goodman M, Kooby DA, Sarmiento JM, Adsay NV: Intraampullary papillary-tubular neoplasm (IAPN): characterization of tumoral intraepithelial neoplasia occurring within the ampulla: a clinicopathologic analysis of 82 cases. Am J Surg Pathol 2010, 34:1731-1748.

6. Kimura W, Futakawa N, Zhao B: Neoplastic diseases of the papilla of Vater. $J$ Hepatobiliary Pancreat Surg 2004, 11:223-231.

7. Morini S, Perrone G, Borzomati D, Vincenzi B, Rabitti C, Righi D, Castri F, Manazza AD, Santini D, Tonini G, Coppola R, Onetti Muda A: Carcinoma of the Ampulla of Vater: Morphological and Immunophenotypical Classification Predicts Overall Survival. Pancreas 2012, 42:60-66.

8. Albores-Saavedra J, Henson DE, Klimstra DS: Tumors of the gallbladder, extrahempatic bile duct and ampulla of Vater. In Atlas of Tumor Pathology. Washington DC: Armed Forces Institute of Pathology; 2000. Fascile 27.

9. Westgaard A, Pomianowska E, Clausen OPF, Gladhaug IP: Intestinal-type and pancreatobiliary-type adenocarcinomas: how does ampullary carcinoma differ from other periampullary malignancies? Ann Surg Oncol 2013, 20:430-439.

10. Pomianowska E, Westgaard A, Mathisen O, Clausen OPF, Gladhaug IP: Prognostic relevance of number and ratio of metastatic lymph nodes in resected pancreatic, ampullary, and distal bile duct carcinomas. Oncol: Ann. Surg; 2012.

11. Riediger H, Keck T, Wellner U, zur Hausen A, Adam U, Hopt UT, Makowiec F: The lymph node ratio is the strongest prognostic factor after resection of pancreatic cancer. J Gastrointest Surg 2009, 13:1337-44.

12. Albores-Saavedra J, Simpson K, Dancer Y-J, Hruban R: Intestinal type adenocarcinoma: a previously unrecognized histologic variant of ductal carcinoma of the pancreas. Ann Diagn Pathol 2007, 11:3-9.

13. Adsay NV, Pierson C, Sarkar F, Abrams J, Weaver D, Conlon KC, Brennan MF, Klimstra DS: Colloid (mucinous noncystic) carcinoma of the pancreas. Am J Surg Pathol 2001, 25:26-42.

14. Adsay NV, Merati K, Basturk O, lacobuzio-Donahue C, Levi E, Cheng JD, Sarkar FH, Hruban RH, Klimstra DS: Pathologically and biologically distinct types of epithelium in intraductal papillary mucinous neoplasms: delineation of an "intestinal" pathway of carcinogenesis in the pancreas. Am J Surg Pathol 2004, 28:839-848.

15. Yopp AC, Katabi N, Janakos M, Klimstra DS, D'Angelica MI, DeMatteo RP, Fong $Y$, Brennan MF, Jarnagin WR, Allen PJ: Invasive carcinoma arising in intraductal papillary mucinous neoplasms of the pancreas: a matched control study with conventional pancreatic ductal adenocarcinoma. Ann Surg 2011, 253:968-974.

16. Waters JA, Schnelldorfer T, Aguilar-Saavedra JR, Chen J-H, Yiannoutsos $C T$, Lillemoe KD, Farnell MB, Sarr MG, Schmidt CM: Survival after resection for invasive intraductal papillary mucinous neoplasm and for pancreatic adenocarcinoma: a multi-institutional comparison according to American Joint Committee on Cancer Stage. J Am Coll Surg 2011, 213:275-283.

17. Zen Y, Quaglia A, Heaton N, Rela M, Portmann B: Two distinct pathways of carcinogenesis in primary sclerosing cholangitis. Histopathology 2011, 59:1100-1110

18. Rocha FG, Lee H, Katabi N, Dematteo RP, Fong Y, D'Angelica Ml, Allen PJ, Klimstra DS, Jarnagin WR: Intraductal papillary neoplasm of the bile duct: a biliary equivalent to intraductal papillary mucinous neoplasm of the pancreas? Hepatology 2012, 56:1352-1360

19. Kohler I, Jacob D, Budzies J, Lehmann A, Weichert W, Schulz S, Neuhaus P, Röcken C: Phenotypic and genotypic characterization of carcinomas of the papilla of Vater has prognostic and putative therapeutic implications. Am J Clin Pathol 2011, 135:202-211.

20. Fischer H-P, Zhou H: Pathogenesis of carcinoma of the papilla of Vater. J Hepatobiliary Pancreat Surg 2004, 11:301-309.

21. Fischer H-P, Zhou H: Pathogenesis and histomorphology of ampullary carcinomas and their precursor lesions. Review and individual findings. Pathologe 2003, 24:196-203.

22. Gassler N, Knüchel R: Tumors of Vater's ampulla. Pathologe 2012, 33:17-23.

23. Verbeke CS, Gladhaug IP: Resection margin involvement and tumour origin in pancreatic head cancer. Br J Surg 2012, 99:1036-1049.

24. Esposito I, Kleeff J, Bergmann F, Reiser C, Herpel E, Friess H, Schirmacher P, Buchler MW: Most pancreatic cancer resections are R1 resections. Ann Surg Oncol 2008, 15:1651-60.

25. Menon KV, Gomez D, Smith AM, Anthoney A, Verbeke CS: Impact of margin status on survival following pancreatoduodenectomy for cancer: the Leeds Pathology Protocol (LEEPP). HPB (Oxford) 2009, 11:18-24.

26. De Jong MC, Tsai $\mathrm{S}$, Cameron JL, Wolfgang CL, Hirose K, van Vledder MG, Eckhauser F, Herman JM, Edil BH, Choti MA, Schulick RD, Pawlik TM: Safety and efficacy of curative intent surgery for peri-ampullary liver metastasis. J Surg Oncol 2010, 102:256-263.

27. Michalski CW, Erkan M, Hüser N, Müller MW, Hartel M, Friess $\mathrm{H}$, Kleeff J: Resection of primary pancreatic cancer and liver metastasis: a systematic review. Dig Surg 2008, 25:473-480.

28. Kang SP, Saif MW: Ampullary and periampullary tumors: translational efforts to meet a challenge in diagnosis and treatment. Highlights from the "2011 ASCO Gastrointestinal Cancers Symposium". San Francisco, CA, USA. January 20-22, 2011. JOP 2011, 12:123-125.

29. Neoptolemos JP, Moore MJ, Cox TF, Valle JW, Palmer DH, McDonald AC, Carter R, Tebbutt NC, Dervenis C, Smith D, Glimelius B, Charnley RM, Lacaine F, Scarfe AG, Middleton MR, Anthoney A, Ghaneh P, Halloran CM, Lerch MM, Oláh A, Rawcliffe CL, Verbeke CS, Campbell F, Büchler MW: Effect of adjuvant chemotherapy with fluorouracil plus folinic acid or gemcitabine vs observation on survival in patients with resected periampullary adenocarcinoma: the ESPAC-3 periampullary cancer randomized trial. JAMA 2012, 308:147-156. 
30. Narang AK, Miller RC, Hsu CC, Bhatia S, Pawlik TM, Laheru D, Hruban RH, Zhou J, Winter JM, Haddock MG, Donohue JH, Schulick RD, Wolfgang CL, Cameron JL, Herman JM: Evaluation of adjuvant chemoradiation therapy for ampullary adenocarcinoma: the Johns Hopkins Hospital-Mayo Clinic collaborative study. Radiat Oncol 2011, 6:126.

31. Palta M, Patel P, Broadwater G, Willett C, Pepek J, Tyler D, Zafar SY, Uronis H, Hurwitz H, White R, Czito B: Carcinoma of the ampulla of Vater: patterns of failure following resection and benefit of chemoradiotherapy. Ann Surg Oncol 2012, 19:1535-1540.

32. Neoptolemos J, European Study Group for Pancreatic Cancer: Ampullary cancer ESPAC-3 (v2) trial: a multicenter, international, open-label, randomized controlled phase III trial of adjuvant chemotherapy versus observation in patients with adenocarcinoma of the ampulla of vater. J Clin Oncol 2011, 29(Suppl), LBA4006.

33. Kim R, Chabot J, Saif MW: Adjuvant treatment for ampullary cancer. Highlights from the "2011 ASCO Annual Meeting". Chicago, IL, USA; June 3-7, 2011. JOP 2011, 12:362-363.

34. Carpelan-Holmstrom M, Nordling S, Pukkala E, Sankila R, Luttges J, Kloppel G, Haglund C: Does anyone survive pancreatic ductal adenocarcinoma? A nationwide study re-evaluating the data of the Finnish Cancer Registry. Gut 2005, 54:385-7.

doi:10.1186/1471-2407-13-428

Cite this article as: Bronsert et al.: Intestinal-type of differentiation predicts favourable overall survival: confirmatory clinicopathological analysis of 198 periampullary adenocarcinomas of pancreatic, biliary, ampullary and duodenal origin. BMC Cancer 2013 13:428.

\section{Submit your next manuscript to BioMed Central and take full advantage of:}

- Convenient online submission

- Thorough peer review

- No space constraints or color figure charges

- Immediate publication on acceptance

- Inclusion in PubMed, CAS, Scopus and Google Scholar

- Research which is freely available for redistribution 